\title{
Periodic modules of large periods for extra-special p-groups
}

\author{
Hiroki SASAKI \\ (Received September 21, 1989)
}

\section{Introduction}

Let $p$ be an odd prime and let $k$ be a field of characteristic $p$. We consider periodic modules over the group algebra of the extra special $p$-group of order $p^{3}$ and of exponent $p$ :

$$
M(p)=\left\langle a, b \mid a^{p}=b^{p}=[a, b]^{p}=1,[a,[a, b]]=[b,[a, b]]=1\right\rangle .
$$

Periodic modules of period $2 p$ had been known only for $M(p)$ by Carlson [6] (1979). In [17] Okuyama and the author gave new examples of such periodic modules for a metacyclic $p$-group

$$
M_{m}(p)=\left\langle a, b \mid a^{p^{m-1}}=b^{p}=1, a^{b}=a^{1+p^{m-2}}\right\rangle, m \geq 3
$$

as the kernels of cocycles representing certatin homogeneous elements of the cohomology algebra $H^{*}\left(M_{m}(p), k\right)$. We note that the maximum possibility of periods of periodic modules over both of the above $p$-groups is $2 p$ by Carlson [7]. In this paper we shall show another examples of periodic $k M(p)$-modules of period $2 p$, which are the kernels of cocycles representing particular homogeneous elements of the cohomology algebra $H^{*}(M(p), k)$.

Before stating our periodic modules we must prepare some notations. Let $G$ be an arbitrary finite group. The cohomology group $H^{r}(G, k)$ is naturally isomorphic to the set of $k G$-homomorphisms of the $r$ th syzygy $\Omega^{r}(k)$ to $k$. Following Carlson, we denote by $\tilde{\xi}$ the $k G$-homomorphism of $\Omega^{r}(k)$ to $k$ corresponding to an element $\varsigma$ in $H^{r}(G, k)$ and let $L_{s}$ denote the kernel of the homomorphism $\hat{\zeta}$. The first cohomology group $H^{1}(G, k)$ is isomorphic with $\operatorname{Hom}(G, k)$. We identify elements in $H^{1}(G$, $k$ ) with those of $\operatorname{Hom}(G, k)$ via this isomorphism. For a normal subgroup $H$ of $G$ of index $p$, the image of an element in $H^{1}(G, k)$ which has $H$ as the kernel under the Bockstein homomorphism of $H^{1}(G, k)$ to $H^{2}$ $(G, k)$ is called a Bockstein element (or a Bockstein for short) corresponding to the subgroup $H$. 
Now let us define our periodic $k M(p)$-module. Let $c=[a, b]$. First of all we note that a Bocksitein corresponding to a maximal subgroup is not zero, because its restriction to another maximal subgroup is a Bockstein corresponding to the subgroup $\langle c\rangle$. Let $\lambda$ and $\mu$ be the elements in $H^{1}(G, k)$ such that

$$
\text { (a) } \lambda=0, \quad \text { (b) } \lambda=1
$$

and

$$
\text { (a) } \mu=1, \quad \text { (b) } \mu=0
$$

and let $\alpha$ and $\beta$ in $H^{2}(M(p), k)$ be the Bocksteins of the element $\lambda$ and $\mu$, respectively. Then $\alpha$ and $\beta$ are Bocksteins corresponding to the maximal subgroups $\langle a, c\rangle$ and $\langle b, c\rangle$, respectively. For a polynomial

$$
f(X)=s_{0}+s_{1} X+\cdots+s_{n-1} X^{n-1}+X^{n}
$$

in $k[X]$ such that

$$
f(i) \neq 0 \text { for } i=0,1, \ldots, p-1,
$$

we define a homogeneous element $\chi$ of degree $2 n$ as follows :

$$
\chi=s_{0} \beta^{n}+s_{1} \alpha \beta^{n-1}+\cdots+s_{n-1} \alpha^{n-1} \beta+\alpha^{n} .
$$

With this notation

THEOREM. If $n \equiv 1$ mod $p$, then the module $L_{x}$ is an indecomposable periodic $k M(p)$-module of period $2 p$.

The periodicity and the indecomposability of the module $L_{x}$ can be shown by using the theory of the module varieties associated with modules, which have been developed by Quillen, Alperin-Evens, Carlson, and Benson, etc.

Let $B$ denote the maximal subgroup $\langle b, c\rangle$ of $M(p)$. Let $\eta$ be the element in $H^{1}(B, k)$ such that $(b) \eta=0$ and $(c) \eta=1$, and let $\xi$ in $H^{2}(B, k)$ be its Bockstein. Let $\tau$ be the image of $\xi$ under the norm map from $H^{2}(B, k)$ to $H^{2 p}(M(p), k)$. In Section 2 we will show the following :

LEMMA 2.1. The tensor product $L_{x} \otimes L_{\tau}$ is a projective $k M(p)$. module. In particular the module $L_{x}$ is periodic.

LEMMA 2.2. The module $L_{x}$ is indecomposable.

In order to determine the period of the module $L_{x}$ we need more information on the cohomology algebra $H^{*}(M(p), k)$. In Section 3 we will 
show a dimension formula :

PROPOSITION 3.1. The element $\tau$ is not a zero-divisor in $H^{*}(M(p), k)$. Moreover the dimension of the cokernel of the homomorphism induced by multiplication by $\tau$ is $2(p+1)$ at each cohomology group $H^{r}(M(p), k)$. In particular one has

$$
\operatorname{dim} H^{r+2 p}(M(p), k)=\operatorname{dim} H^{r}(M(p), k)+2(p+1) \text { for } r \geqq 0 .
$$

This will be established by investigating the cohomology exact sequences associated with the extension which corresponds to the norm $\tau$. The extension we deal with is the $\bmod p$ version of that of $\boldsymbol{Z} M(p)$-lattices in the section 6 in Lewis [15], where some homomorphisms of the integral cohomology groups associated with the extension was considered. Also shown in Section 3 is

LEMMA 3.3. The second cohomology group $H^{2}(M(p), k)$ is four dimensional.

Using these facts we will verify that the period of the module $L_{x}$ is in fact $2 p$ if $n \equiv 1 \bmod p$ in Section 4 .

If the underlying field $k$ is not the prime field, then one can take a $\chi$ in $H^{2}(M(p), k)$. Therefore Lemma 2.1 and Proposition 3.1 together with Lemma 3.1 in Okuyama-Sasaki [17] imply that the cohomology algebra $H^{*}(M(p), k)$ is generated by $\chi, \tau$, and $\sum_{i=0}^{2 p} H^{i}(M(p), k)$. We believe that this is useful to determine the cohomology algebra.

All modules considered are finitely generated right modules. Maps are written on the right with the convention of writing composites.

We fix some more notations. Let $G$ be a finite group. The restriction of a $k G$-module $M$ to a subgroup $H$ of $G$ is denoted $M_{\mid H}$. For $\phi$ a $k G$-homomorphism of $k G$-modules we denote by $\phi_{\mid H}$ the restriction of $\phi$ to $H$. If $\gamma$ is an element in $H^{r}(G, k)$, then $\gamma_{H}$ is the restriction of $\gamma$ to $H$. And for $\delta$ an element in $H^{r}(H, k)$ we denote by $\delta^{\otimes G}$ the image of $\delta$ under the norm map from $H^{r}(H, k)$ to $H^{r|G: H|}(G, k)$. The restriction map from $H^{r}(G, M)$ to $H^{r}(H, M)$ is denoted by $\operatorname{res}_{H}^{G}$. If there is no fear of confusion we omit the superscript $G$. The corestriction map from $H^{r}(H, M)$ to $H^{r}(G, M)$ is denoted by $\operatorname{cor}_{H}^{G}$. We omit the subscript $H$ if there is no fear of confusion.

Henceforth let $G$ denote the $p$-group $M(p)$ unless otherwise stated.

\section{The periodicity and the indecomposability}

In this section we shall prove that the module $L_{x}$ is a periodic 
indecomposable $k M(p)$-module. We use the theory of the cohomology varieties associated with modules. For the convenience of the reader we write down the definition and some results we need here.

For $G$ a finite group let $H(G)=\sum_{i \geq 0} H^{2 i}(G, k)$. If $M$ is a $k G$-module, then we let

$$
r_{G}(M)=\operatorname{rad} \operatorname{ann}_{H(G)} H^{*}(G, M \otimes S),
$$

where $S$ is the direct sum of the simple $k G$-modules one for each isomorphism classes. The cohomology variety $X_{G}(M)$ associated with the module $M$ is defined to be the prime spectrum which consists of the prime ideals containing $r_{G}(M)$ :

$$
X_{G}(M)=\operatorname{Spec}\left(H(G), r_{G}(M)\right) .
$$

The module $M$ is projective if and only if the variety $X_{G}(M)$ consists of only the irrelevant maximal ideal $H^{+}(G)=\sum_{i \geq 1} H^{2 i}(G, k)$. The fundamental theorem is the following :

THEOREM (Alperin-Evens). With the same notation as above it follows that

$$
r_{G}(M)=\cap \operatorname{res}_{E}^{-1} r_{E}\left(M_{\mid E}\right),
$$

where $E$ runs over all elementary abelian p-subgroups of $G$.

Chouinard's theorem follows from the above theorem. The following lemma is obtained by mainly J. Carlson, See [8], [9], and [10], or Benson [3].

Lemma. (1) Let $M$ and $N$ be kG-modules. Then

$$
X_{G}(M \otimes N)=X_{G}(M) \cap X_{G}(N) .
$$

(2) For $\gamma$ an element in $H^{r}(G, k)$, one has

$$
X_{G}\left(L_{\gamma}\right)=X_{G}(\gamma) \text {. }
$$

Namely $r_{G}\left(L_{\gamma}\right)=\operatorname{rad}(\gamma)$. The module $L_{\gamma}$ is indecomposable if and only if $r_{G}(L \gamma)$ is a prime ideal.

Now let us proceed to our argument.

LEMMA 2.1. The tensor product $L_{x} \otimes L_{\tau}$ is a projective $k M(p)$. module. In particular the module $L_{x}$ is periodic.

PROOF. It is sufficient to show that the restriction of $L_{x} \otimes L_{\bar{t}}$ to every maximal elementary abelian subgroup of $G$ is projective. One has for $H$ 
a subgroup of $G$

$$
\left(L_{\chi} \otimes L_{\tau}\right)_{\mid H} \simeq L_{\left(\chi_{\mid H)}\right.} \otimes L_{\left(\tau_{\mid H}\right)} \oplus(\text { projective }) .
$$

Hence we see that

$$
X_{H}\left(L_{\left(\chi_{\mid H}\right)} \otimes L_{\left(\tau_{\mid H}\right)}\right)=X_{H}\left(\chi_{\mid H}, \tau_{\mid H}\right) .
$$

Recall that

$$
\chi=\mathrm{s}_{0} \beta^{n}+\mathrm{s}_{1} \alpha \beta^{n-1}+\cdots+\mathrm{s}_{n-1} \alpha^{n-1} \beta+\alpha^{n} .
$$

First we consider about restriction to the subgroups $\left\langle a b^{i}, c\right\rangle_{\mathrm{s}}$. For $i$, $0 \leq i \leq p-1$, let $A_{i}=\left\langle a b^{i}, c\right\rangle$ and $\xi_{i}$ be a Bockstein in $H^{2}\left(A_{i}, k\right)$ corresponding to the subgroup $\left\langle a b^{i}\right\rangle$. And put $\varsigma_{i}=\beta_{\mid A_{i}}$. Then $\varsigma_{i}$ is a Bockstein in $H^{2}\left(A_{i}, k\right)$ corresponding to the subgroup $\langle c\rangle$. Since $(\alpha-i \beta)_{\mid A_{i}}=0$, we have $\alpha_{\mid A_{i}}=i_{\zeta_{i}}$, so that

$$
\begin{aligned}
\chi_{\mid A_{i}} & =s_{0} \varsigma_{i}^{n}+s_{1} i_{\varsigma_{i}^{n}}+\cdots+s_{n-1} i^{n-1} \varsigma_{i}^{n}+i^{n} \varsigma_{i}^{n} \\
& =f(i) \varsigma_{i}^{n} .
\end{aligned}
$$

On the other hand by Mackey formula for the norm map we get

$$
\begin{aligned}
\tau_{\mid A_{i}} & =\xi^{\otimes G_{\mid A_{i}}} \\
& =\left(\xi_{\mid B \cap A_{i}}\right)^{\otimes A_{i}} \\
& =(\xi \mid\langle c\rangle)^{\otimes A_{i}} .
\end{aligned}
$$

Since the Bockstein $\xi$ in $H^{2}(B, k)$ corresponds to the subgroup $\langle b\rangle$, its restriction to the subgroup $\langle c\rangle$ is not zero. Therefore we have, by Lemma 3. 1 in Okuyama-Sasaki [16],

$$
\left(\xi_{\mid\langle c\rangle}\right)^{\otimes A_{i}}=\xi_{i}^{p}-\varsigma_{i}^{p-1} \xi_{i}
$$

and so

$$
\tau_{A i}=\xi_{i}^{p}-\varsigma_{i}^{p-1} \xi_{i}
$$

Consequently we get

$$
\begin{aligned}
X_{A i}\left(L_{\chi} \otimes L_{\tau}\right) & =X_{A_{i}}\left(\varsigma_{i}, \xi_{i}\right) \\
& =\{0\} .
\end{aligned}
$$

Namely the restriction of the tensor product $L_{\chi} \otimes L_{\tau}$ to the subgroup $A_{i}$ is projective, $i=0,1, \ldots, p-1$.

Next we deal with the subgroup $B$. Let $\varsigma=\alpha_{\mid B}$. Then $\varsigma$ is a Bockstein in $H^{2}(B, k)$ corresponding to the subgroup $\langle c\rangle$. Since $\beta_{\mid B}=0$, we have 


$$
\begin{aligned}
\chi_{\mid B} & =\left(\alpha_{\mid B}\right)^{n} \\
& =\varsigma^{n} .
\end{aligned}
$$

On the other hand by Mackey formula we get

$$
\begin{aligned}
\tau_{\mid B} & =\xi^{\otimes G}{ }_{\mid B} \\
& =\prod_{i=0}^{p-1} \xi^{a^{i}} \\
& =\prod_{i=0}^{p-1}\left(\xi+i_{\varsigma}\right) \\
& =\xi^{p}-\varsigma^{p-1} \xi,
\end{aligned}
$$

because $\xi^{a}=\varsigma+\xi$. Similarly to the former case, we see that the tensor product $L_{\chi} \otimes L_{\tau}$ is projective over $k B$.

Thus the tensor product $L_{\chi} \otimes L_{\tau}$ is a projective module. By the argument in the proof of Theorem 8.7 in Carlson [10] the module $L_{\chi}$ is periodic. This completes the proof of the lemma.

LEMMA 2.2. The module $L_{\chi}$ is indecomposable.

PROOF. By Lemma (2) it is enough to prove that the radical of the principal ideal $(\boldsymbol{\chi})$ in $H(G)$ is a homogeneous prime ideal. We use the same notations as in the proof of the previous lemma. Since the radical of the principal ideal $(\varsigma)$ in $H(B)$ is a homogeneous prime ideal, its inverse image $\operatorname{res}_{B}^{-1}(\operatorname{rad}(\varsigma))$ is a homogeneous prime ideal in $H(G)$. We shall show that

$$
\operatorname{rad}(\chi)=\operatorname{res}_{B}^{-1}(\operatorname{rad}(\varsigma))
$$

which proves the lemma.

Since $\chi_{\mid B}=\varsigma^{n}$, it follows that

$$
\operatorname{rad}(\chi) \subset \operatorname{res}_{B}^{-1}(\operatorname{rad}(\varsigma))
$$

Next for an element $\sigma$ in $\operatorname{res}_{B}^{-1}(\operatorname{rad}(\varsigma))$ we can choose a number $m$ such that

$$
\left(\sigma_{\mid B}\right)^{m}=\alpha_{\mid B} h(\alpha, \tau)_{\mid B}
$$

for some polynomial $h(X, Y)$ in $k[X, Y]$, because $\sigma_{\mid B}$ is $G$-invariant and a non-nilpotent $G$-invariant element in $H(B)$ is a polynomial in $\alpha_{\mid B}$ and $\tau_{\mid B}$. By Lemma in Quillen-Venkov [19] the square $\left(\sigma^{m}-\alpha h(\alpha, \tau)\right)^{2}$ is contained in the ideal $(\beta)$. Thus the element $\sigma$ is contained in the radical of the ideal $(\alpha, \beta)$. Namely we have

$$
\operatorname{res}_{B}^{-1}(\operatorname{rad}(\varsigma)) \subset \operatorname{rad}(\alpha, \beta) .
$$


Finally by a theorem of Serre [21] it follows that

$$
\left(\prod_{i=0}^{p-1}(\alpha-i \beta)\right) \beta=0 \text {. }
$$

Hence a minimal prime divisor $p$ of $(\chi)$ contains either one of the $(\alpha-i \beta) \mathrm{s}, 0 \leq i \leq p-1$, or $\beta$. If $\beta$ is contained in $\mathfrak{p}$, then so is the element $\alpha$, beoanse $\chi \equiv 0 \bmod \mathfrak{p}$. If $\alpha-i \beta$ is contained in $\mathfrak{p}$, then we have

$$
\begin{aligned}
0 & \equiv s_{0} \beta^{n}+s_{1} i \beta^{n}+\cdots+s_{n-1} i^{n-1} \beta^{n}+i^{n} \beta^{n} \\
& =f(i) \beta^{n} \bmod \mathfrak{p} .
\end{aligned}
$$

Both $\alpha$ and $\beta$ are therefore contained in the prime ideal $\mathfrak{p}$ also in this case. Thus the elements $\alpha$ and $\beta$ are contained in the radical of the ideal $(\chi)$. Namely we get

$$
\operatorname{rad}(\alpha, \beta) \subset \operatorname{rad}(\chi) .
$$

Consequently we obtain the equality

$$
\operatorname{rad}(\chi)=\operatorname{res}_{B}^{-1}(\operatorname{rad}(\varsigma)),
$$

as desired.

REMARK. Serre's theorem was also proved by Okuyama-Sasaki [16].

\section{A dimension formula}

The Bockstein element $\xi$ in $H^{2}(B, k)$ corresponding to the subgroup $\langle b\rangle$ corresponds to the following extension of $k B$-modules :

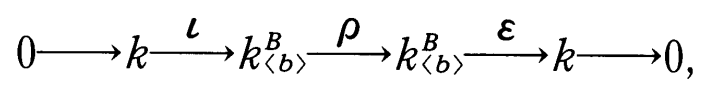

where

$$
\begin{aligned}
& \iota: k \longrightarrow k_{\langle b\rangle}^{B} ; 1 \longmapsto 1 \otimes(c-1)^{p-1} \\
& \rho: k\langle b\rangle \longrightarrow k_{\langle b\rangle}^{B} ; 1 \otimes 1 \longmapsto 1 \otimes(c-1)
\end{aligned}
$$

and

$$
\varepsilon: k_{\langle b\rangle}^{B} \longrightarrow k ; 1 \otimes 1 \longmapsto 1 .
$$

Regarding $k \stackrel{\iota}{\longrightarrow} k_{\langle b\rangle}^{B} \stackrel{\rho}{\longrightarrow} k_{\langle b\rangle}^{B}$ as a complex of $k B$-modules, we form its tensor induction to $G$ (see Evens [13]) :

$$
E_{2 p} \longrightarrow E_{2 p-1} \longrightarrow E_{2 p-2} \longrightarrow \cdots \longrightarrow E_{j} \longrightarrow \cdots \longrightarrow E_{1} \longrightarrow E_{0}
$$

Then the extension 


$$
0 \longrightarrow k \stackrel{\partial_{2 p}}{\longrightarrow} E_{2 p-1} \stackrel{\partial_{2 p-1}}{\longrightarrow} E_{2 p-2} \longrightarrow \cdots \stackrel{\partial_{j+1}}{\longrightarrow} E_{j} \longrightarrow \cdots \longrightarrow E_{1} \longrightarrow E_{0} \stackrel{\partial_{0}}{\longrightarrow} k \longrightarrow 0
$$

corresponds to the norm $\tau=\xi^{\otimes G}$ and each term satisfies the following :

$$
\begin{gathered}
E_{0} \simeq \bigoplus_{i=0}^{p-1} k_{\left\langle a b^{i}\right\rangle}^{G} \oplus \text { (projective) } \\
E_{p} \simeq \bigoplus_{i=0}^{p-1} k_{\left\langle a b^{i}\right\rangle}^{G} \oplus \text { (projective) } \\
E_{2 p-2} \simeq k_{\langle b\rangle}^{G} \oplus \text { (projective) } \\
E_{2 p-1} \simeq k_{\langle b\rangle}^{G}
\end{gathered}
$$

and

other $E_{j} \mathrm{~s}$ are projective.

This can be verified as in Section 6 of Lewis [15], so that we omit the proof. Our aim in this section is to prove the following:

PROPOSITION 3.1. The element $\tau$ is not a zero-divisor in $H^{*}(M(p), k)$. Moreover the dimension of the cokernel of the homomorphism induced by multiplication by $\tau$ is $2(p+1)$ at each cohomology group $H^{r}(M(p), k)$. In particular one has

$$
\operatorname{dim} H^{r+2 p}(M(p), k)=\operatorname{dim} H^{r}(M(p), k)+2(p+1) \text { for } \mathrm{r} \geq 0 .
$$

To prove this proposition we need the following lemma.

LEMMA 3.2. Let $E=\langle x, y\rangle$ be an elementary abelian p-group of order $p^{2}, p$ an odd prime. Then the homomorphism

$$
\bigoplus_{i=0}^{p-1} \mathrm{res}\left\langle x y_{i}\right\rangle: H^{r}(E, k) \longrightarrow \bigoplus_{i=0}^{p-1} H^{r}\left(\left\langle x y^{i}\right\rangle, k\right)
$$

is epimorphic when $r \geq 2 p-3$.

PROOF. Let us denote by RES the above homomorphism. Let $\lambda_{x}$ and $\lambda_{y}$ be the elements in $H^{1}(E, k)$ such that

$$
(x) \lambda_{x}=0,(y) \lambda_{x}=1 \text { and }(x) \lambda_{y}=1,(y) \lambda_{y}=0,
$$

and let $\beta_{x}$ and $\beta_{y}$ be the Bocksteins of $\lambda_{x}$ and $\lambda_{y}$, respectively. Then one has

$$
H^{*}(E, k)=k\left[\beta_{x}, \beta_{y}\right] \otimes \Lambda\left(\lambda_{x}, \lambda_{y}\right) .
$$

Let $\eta_{i}$ be the element in $H^{1}\left(\left\langle x y^{i}\right\rangle, k\right)$ such that

$$
\left(x y^{i}\right) \eta_{i}=1
$$

and let $\varsigma_{i}$ in $H^{2}\left(\left\langle x y^{i}\right\rangle, k\right)$ be its Bockstein, $i=0,1, \cdots, p-1$. Then one 
has

$$
H^{*}\left(\left\langle x y^{i}\right\rangle, k\right)=k\left[\varsigma_{i}\right] \otimes \Lambda\left(\eta_{i}\right) .
$$

These elements satisfy the following relations:

$$
\lambda_{\left.\left.x\right|_{<x y i}\right\rangle}=i \eta_{i}, \lambda_{\left.\left.y\right|_{<x y i}\right\rangle}=\eta_{i}
$$

and

$$
\beta_{x \mid<x y i}=i_{\varsigma i}, \beta_{y|<x y i\rangle}=\varsigma_{i}
$$

When $r$ is even we set $r=2 s$. We can take a set $\left\{\beta_{y}^{s}, \beta_{x} \beta_{y}^{s-1}, \cdots, \beta_{x}^{s}\right.$, $\left.\beta_{y}^{s-1} \lambda_{x} \lambda_{y}, \beta_{x} \beta_{y}^{s-2} \lambda_{x} \lambda_{y}, \cdots, \beta_{x}^{s-1} \lambda_{x} \lambda_{y}\right\}$ as a basis of $H^{2 s}(E, k)$. And we take the basis $\left\{\varsigma^{s}\right\}$ of $H^{2 s}\left(\left\langle x y^{i}\right\rangle, k\right)$. With respect to these bases the homomorphism RES is represented by the following matrix :

$$
\left[\begin{array}{cccccc}
1 & 1 & \cdots & 1 & \cdots & 1 \\
0 & 1 & \cdots & i & \cdots & p-1 \\
& & & \cdots \cdots \ldots \ldots . . & & \\
0 & 1 & \cdots & i^{j} & \cdots & (p-1)^{j} \\
& & & \cdots \ldots \ldots \ldots & & \\
0 & 1 & \cdots & i^{s} & \cdots & (p-1)^{s} \\
0 & 0 & \cdots & 0 & \cdots & 0 \\
& & & \cdots \cdots \cdots \cdots & & \\
0 & 0 & \cdots & 0 & \cdots & 0
\end{array}\right]
$$

The rank of this matrix is $p$ if $s \geq p-1$ and $s+1$ otherwise.

When $r$ is odd, we set $r=2 s+1$. With respect to the bases $\left\{\beta_{y}^{s} \lambda_{x}\right.$, $\left.\beta_{x} \beta_{y}^{s-1} \lambda_{x}, \cdots, \beta_{x}^{s} \lambda_{x}, \beta_{y}^{s} \lambda_{y}, \beta_{x} \beta_{y}^{s-1} \lambda_{y}, \cdots, \beta_{x}^{s} \lambda_{y}\right\}$ of $H^{2 s+1}(E, k)$ and $\left\{s_{i}^{s} \eta_{i}\right\}$ of $H^{2 s+1}\left(\left\langle x y^{i}\right\rangle, k\right)$ the homomorphism RES is represented by the following matrix :

$$
\left[\begin{array}{cccccc}
0 & 1 & \cdots & i & \cdots & p-1 \\
& & & \cdots \cdots \cdots \cdots & & \\
0 & 1 & \cdots & i^{j+1} & \cdots & (p-1)^{j+1} \\
& & & \cdots \cdots \cdots \cdots & & \\
0 & 1 & \cdots & i^{s+1} & \cdots & (p-1)^{s+1} \\
1 & 1 & \cdots & 1 & \cdots & 1 \\
& & & \cdots \cdots \cdots \cdots & & \\
0 & 1 & \cdots & i^{j} & \cdots & (p-1)^{j} \\
& & & \cdots \cdots \cdots \cdots & & \\
0 & 1 & \cdots & i^{s} & \cdots & (p-1)^{s}
\end{array}\right]
$$


The rank of this matrix is $p$ if $s \geq p-2$ and $s+2$ otherwise. This completes the proof of the lemma.

Proof of Proposition 3.1. Let $K_{i+1}=\operatorname{Ker} \partial_{i}, i=0,1, \cdots, 2 p-2$. The extension is decomposed into $2 p$ short exact sequences :

$$
\begin{aligned}
& 0 \longrightarrow K_{1} \stackrel{\varkappa_{0}}{\longrightarrow} E_{0} \stackrel{\partial_{0}}{\longrightarrow} k \longrightarrow 0 \\
& 0 \longrightarrow K_{i+1} \stackrel{\varkappa_{i}}{\longrightarrow} E_{i} \stackrel{\partial_{i}}{\longrightarrow} K_{i} \longrightarrow 0,1 \leq_{i} \leq 2 p-2 \\
& 0 \longrightarrow k \stackrel{\varkappa_{2 p-1}}{\longrightarrow} E_{2 p-1} \stackrel{\partial_{2 p-1}}{\longrightarrow} K_{2 p-1} \longrightarrow 0 .
\end{aligned}
$$

Associated with these short exact sequences there are $2 p$ connecting homomorphisms :

$$
\begin{aligned}
& \omega_{0}: H^{r}(G, k) \longrightarrow H^{r+1}\left(G, K_{1}\right) \\
& \omega_{i}: H^{r+i}\left(G, K_{i}\right) \longrightarrow H^{r+i+1}\left(G, K_{i+1}\right), 1 \leq i \leq 2 \mathrm{p}-2 \\
& \omega_{2 p-1}: H^{r+2 p-1}\left(G, K_{2 p-1}\right) \longrightarrow H^{r+2 p}(G, k) .
\end{aligned}
$$

The composition map of these connecting homomorphisms is exactly the homomorphism induced by multiplication by the element $\tau$. Unless $j=0$, $p, 2 p-2$, and $2 p-1$, the connecting homomorphism $\omega_{j}$ is isomorphic, for the module $E_{j}$ is projective. In what follows we shall show that the others are all monomorphic so that the dimension of the cokernel of the homomorphism $(\cdot \tau)$ is the sum of those of the connecting homomorphisms. When we deal with one connecting homomorphism $\omega_{j}$ we will omit the index $j$ from the notations $\varkappa_{j}$ and $\partial_{j}$. We shall use the theory of relative projective covers. See Knörr [14] for relative projective covers.

STEP 1. The connecting homomorphism wo is monomorphic and the dimension of its cokernel is $p$.

PROOF. First we show that the induced homomorphism $\partial_{0 *}$ is the zero homomorphism in the exact cohomology sequence

$$
\begin{aligned}
\cdots \longrightarrow H^{r}\left(G, K_{1}\right) \stackrel{\varkappa_{0 *}}{\longrightarrow} H^{r}\left(G, E_{0}\right) & \\
& \stackrel{\partial_{0 *}}{\longrightarrow} H^{r}(G, k) \stackrel{\omega_{0}}{\longrightarrow} H^{r+1}\left(G, K_{1}\right) \longrightarrow \cdots .
\end{aligned}
$$

Recall that

$$
E_{0} \simeq \bigoplus_{i=0}^{p-1} k_{\left\langle a b^{i}\right\rangle}^{G} \oplus \text { (projective) }
$$

Let $\nu_{i}: k_{\left\langle a b^{i}\right\rangle}^{G} \longrightarrow E_{0}$ be the injection with respect to the above decomposition and let $\delta_{i}=\nu_{i} \partial: k_{\left\{a b^{i}\right\rangle}^{G} \longrightarrow k, 0 \leq i \leq p-1$. Then it follows that 


$$
\partial_{*}=\sum_{i=0}^{p-1} \delta_{i} *
$$

By our construction of the module $E_{0}$ and the homomorphism $\partial_{0}$, one can verify that each homomorphism $\delta_{i}$ is not the zero homomorphism. In particular the module $E_{0}$ is the direct sum of the relative $\left\{\left\langle a b^{i}\right\rangle \mid i=0, \cdots\right.$, $p-1\}$-projective cover of the trivial module $k$ and a projective module. Hence we have the following commutative diagram:

$$
\bigoplus_{i=0}^{p-1} H^{r}\left(G, k_{\left\langle a b^{i}\right\rangle}^{G}\right) \stackrel{\sum_{i=0}^{p-1} \delta_{i *}}{\longrightarrow} H^{r}(G, k)
$$

$$
\begin{aligned}
& \text { Eckmann-Shapiro \| } \\
& \qquad \bigoplus_{i=0}^{p-1} H^{r}\left(\left\langle a b^{i}\right\rangle, k\right) \underset{\sum_{i=0}^{p-1} \operatorname{cor}\left\langle a b^{i}\right\rangle}{\stackrel{p-1}{\sim} \underset{i=0}{\longrightarrow} \bigoplus_{i=0}^{p-1} H^{r}\left(\left\langle a b^{i}\right\rangle, k\right)}
\end{aligned}
$$

where $\varepsilon_{i}: k \longrightarrow k$ is the homomorphism corresponding to $\delta_{i}$ under the isomorphism of $\operatorname{Hom}_{k G}\left(k_{\left\langle a b^{i}\right\rangle}^{G}, k\right)$ to $\operatorname{Hom}_{k\left\langle a b^{i}\right\rangle}(k, k)$. But since the subgroup $A_{i}$ is abelian it follows that

$$
\begin{aligned}
\operatorname{cor}_{\left\langle a b^{i}\right\rangle}^{G} & =\operatorname{cor}_{\langle a b i\rangle}^{A_{i}} \operatorname{cor}_{A_{i}}^{G} \\
& =0,
\end{aligned}
$$

and so we get

$$
\partial_{*}=0 \text {. }
$$

Thus we obtain the exact sequence

$$
0 \longrightarrow H^{r}(G, k) \stackrel{\omega_{0}}{\longrightarrow} H^{r+1}\left(G, K_{1}\right) \stackrel{\mathcal{K}_{*}}{\longrightarrow} H^{r+1}\left(G, E_{0}\right) \longrightarrow 0,
$$

therefore

$$
\begin{aligned}
\operatorname{dim} \text { Coker } \omega_{0} & =\operatorname{dim} H_{p-1}^{r+1}\left(G, E_{0}\right) \\
& =\operatorname{dim} \bigoplus_{i=0}^{r+1} H^{r+1}\left(\left\langle a b^{i}\right\rangle, k\right) \\
& =p,
\end{aligned}
$$

as desired.

STEP 2. The connecting homomorphism $\omega_{p}$ is monomorphic and the dimension of its cokernel is $p$.

PROOF. Recall that 


$$
E_{p} \simeq \bigoplus_{i=0}^{p-1} k_{\langle a b i\rangle}^{G} \oplus P,
$$

where $P$ is a projective $k G$-module. Similarly to Step 1 it is enough to show that the induced homomorphism $\partial_{*}$ is the zero homomorphism in the exact cohomology sequence

$$
\begin{aligned}
& \cdots \longrightarrow H^{r+p}\left(G, K_{p+1}\right) \stackrel{\mathcal{\varkappa}_{*}}{\longrightarrow} H^{r+p}\left(G, E_{p}\right) \stackrel{\partial_{*}}{\longrightarrow} H^{r+p}\left(G, K_{p}\right) \\
& \stackrel{\omega_{p}}{\longrightarrow} H^{r+p+1}\left(G, K_{p+1}\right) \longrightarrow \cdots .
\end{aligned}
$$

Let $\phi_{i}$ be the projection of $E_{p}$ to $k_{\langle a b i\rangle}^{G}$ and $\nu_{i}$ be the injection of $k_{\left\langle a b^{i}\right\rangle}^{G}$ to $E_{p}$, respectively, with respect to the direct decomposition above, $0 \leq i \leq$ $p-1$. Then the homomorphism $\varkappa \phi_{i}: K_{p+1} \longrightarrow k_{\left\{a b^{i}\right\rangle}^{G}$ is not the zero homorphism. For otherwise the induced module $k_{\langle a b i\rangle}^{G}$ is a direct summand of the module $K_{p}$ so that the module $K_{1}$ has a $\left\langle a b^{i}\right\rangle$-projective direct summand, since the modules $E_{p-1}, \cdots, E_{1}$ are all projective. But the module $K_{1}$ has no such direct summand, since the module $E_{0}$ is the direct sum of $\left\{\left\langle a b^{i}\right\rangle \mid i=0, \cdots, p-1\right\}$-projective cover of the trivial module $k$ and a projective module. We set $\delta_{i}=\nu_{i} \partial: k_{\left\langle a b^{i}\right\rangle}^{G} \longrightarrow K_{p}$. As in the step 1, it is sufficient to show that $\delta_{i *}=0$ for $i=0, \cdots, p-1$. Let

$$
\pi_{i}: k_{\left\langle a b^{i}\right\rangle}^{G} \longrightarrow k_{\left\langle a b^{i}\right\rangle}^{\left.A_{i}\right\rangle} \text { and } \theta_{i}: k_{\left\langle a b^{i}\right\rangle}^{\left.A_{i}\right\rangle} \longrightarrow k_{\left\langle a b^{i}\right\rangle}^{G}
$$

be the projection and the injection with respect to the $k A_{i}$-decomposition

$$
k_{\left\langle a b^{i}\right\rangle \mid A_{i}}^{G}=k_{\left\langle a b^{i}\right\rangle}^{A_{i}} \oplus \sum_{1 \neq t \in A_{i} \backslash G} k_{\left\langle a b^{i} i\right\rangle}^{A_{i}} \otimes t .
$$

Then the homomorphism

$$
\theta_{i}^{*}: \operatorname{Hom}_{k G}\left(k_{\left\langle a b^{i}\right\rangle}^{G}, K_{p}\right) \longrightarrow \operatorname{Hom}_{k A_{i}}\left(k_{\left\langle a b^{i}\right\rangle}^{\left.A_{i}\right\rangle}, K_{p}\right)
$$

is isomorphic. Let $\psi_{i}=\theta_{i}\left(\delta_{i \mid A_{i}}\right)$. We obtain the following commutative diagram :

$$
\begin{aligned}
& H^{r+p}\left(G, k_{\left\langle a b^{i}\right\rangle}^{G}\right) \stackrel{\delta_{i *}}{\longrightarrow} H^{r+p}\left(A_{i}, k_{\left\langle a b^{i}\right\rangle}^{G}\right) \\
& \operatorname{res}_{A_{i}} \prec \quad \text { ॥ } E-S \quad \uparrow \operatorname{cor}^{G} \\
& H^{r+p}\left(A_{i}, k_{\left\langle a b^{i}\right\rangle}^{G}\right) \stackrel{\pi_{i *}}{\longrightarrow} H^{r+p}\left(A_{i}, k_{\left\langle a b^{i}\right\rangle}^{A_{i}}\right) \stackrel{\psi_{i *}}{\longrightarrow} H^{r+p}\left(A_{i}, K_{p}\right) \\
& \theta_{i *} \uparrow \quad \nearrow \quad\left(\delta_{i \mid A_{i}}\right)_{*} \\
& H^{r+p}\left(A_{i}, k_{\left\langle a b^{i}\right\rangle}^{G}\right)
\end{aligned}
$$


where "E-S" means "Eckmann-Shapiro". Namely we have

$$
\begin{aligned}
\delta_{i *} & =\operatorname{res}_{A_{i}} \pi_{i} \psi_{i *} \operatorname{cor}^{G} \\
& =\operatorname{res}_{A_{i}} \pi_{i *} \theta_{i *}\left(\delta_{i \mid A_{i}}\right)_{*} \operatorname{cor}^{G} .
\end{aligned}
$$

Thus it is enough to prove that

$$
\left(\delta_{i \mid A_{i}}\right)_{*}=0 .
$$

Now since $E_{2 p-1}, E_{2 p-2}, \cdots, E_{p+1}$ are projective over $k A_{i}$, we see that

$$
K_{p+1 \mid A_{i}} \simeq \Omega^{-(p-1)}\left(k_{A_{i}}\right) \oplus \text { (projective) }
$$

and

$$
k_{\left\langle a b^{i}\right\rangle \mid A_{i}}^{G} \simeq \bigoplus_{j=0}^{p-1} k_{\left\langle a b^{i} c^{j}\right\rangle}^{A_{i}} .
$$

These together with the fact that the homomorphism $\varkappa \phi_{i}$ is not the zero homomorphism for each $i=0, \cdots, p-1$ give the following commutative diagram:

$$
\begin{aligned}
& H^{r+p}\left(A_{i}, K_{p+1}\right) \stackrel{\left(\varkappa \phi_{i \mid A_{i}}\right)_{*}}{\longrightarrow} H^{r+p}\left(A_{i}, \bigoplus_{j=0}^{p-1} k_{\left\langle a b^{i} c^{j}\right\rangle}^{A_{i}}\right) \\
& \text { २। } \quad \text { । dimension shifting } \\
& H^{r+2 p-1}\left(A_{i}, k\right) \stackrel{\eta *}{\longrightarrow} H^{r+2 p-1}\left(A_{i}, \bigoplus_{j=0}^{p-1} k_{\left\langle a b^{i} c^{j}\right\rangle}^{A_{i}}\right) \\
& \bigoplus_{j=0}^{p-1} \mathrm{res}\left\langle a b^{i} c^{j}\right\rangle \mid \text { Eckmann-Shapiro } \\
& \bigoplus_{j=0}^{p-1} H^{r+2 p-1}\left(\left\langle a b^{i} c^{j}\right\rangle, k\right) \underset{\nu *}{\sim} \underset{j=0}{\sim} \bigoplus_{j-1}^{r+2 p-1}\left(\left\langle a b^{i} c^{j}\right\rangle, k\right)
\end{aligned}
$$

where the homomorphism $\eta: k \longrightarrow \bigoplus_{j=0}^{p-1} k_{\left\langle a b^{i} c^{i}\right\rangle}^{\left.A_{i}\right\rangle}$ is a $k A_{i}$-homomorphism which is determined by the homomorphism $\varkappa \phi_{i \mid A_{i}}$ of $K_{p+1}$ to $\bigoplus_{j=0}^{p-1} k_{\left\langle a b^{i} c^{j}\right\rangle}^{A_{i}}$ through projective resolutions, and the homomorphism $\nu$ is the homomorphism which corresponds to $\eta$ under the isomorphism of $\operatorname{Hom}_{k A_{i}}\left(k, \bigoplus_{j=0}^{p-1}\right.$ $k_{\left\langle a b^{i} c^{j}\right\rangle}^{\left.A_{i}\right\rangle}$ to $\bigoplus_{j=0}^{p-1} \operatorname{Hom}_{k\left\langle a b^{i} c^{j}\right\rangle}(k, k)$. The homomorphism $\left(\varkappa \phi_{i \mid A_{i}}\right)_{*}$ is epimorphic, because so is the homomorphism $\bigoplus_{j=0}^{p-1}$ res $\left\langle a b^{i} c^{j}\right\rangle$ by Lemma 3.1. Namely $H^{r+p}\left(A_{i}, k_{\left\langle a b^{i}\right\rangle}^{G}\right)\left(\nu_{i \mid A_{i}}\right)_{*}$ is contained in the kernel of $\left(\partial_{\mid A i}\right)_{*}$. Consequently we have

$$
\left(\delta_{i \mid A_{i}}\right)_{*}=0,
$$


as desired.

STEP 3. The connecting homomorphism $\omega_{2 p-2}$ is monomorphic and the dimension of its cokernel is 1.

PROOF. Recall that

$$
E_{2 p-2} \simeq k_{\langle b\rangle}^{G} \oplus P
$$

where $P$ is a projective $k G$-module. It is enough to show that the induced homomorphism $\partial_{*}$ is the zero homomorphism in the exact cohomology sequence

$$
\begin{aligned}
\stackrel{\partial_{*}}{\longrightarrow} H^{r+2 p-2}\left(G, K_{2 p-1}\right) \stackrel{\mathcal{K} *}{\longrightarrow} H^{r+2 p-2}\left(G, E_{2 p-2}\right) \\
\stackrel{\partial_{*}}{\longrightarrow} H^{r+2 p-2}\left(G, K_{2 p-1}\right) \stackrel{\omega_{2 p-2}}{\longrightarrow} H^{r+2 p-1}\left(G, K_{2 p-1}\right) \longrightarrow \cdots .
\end{aligned}
$$

Let $\nu$ be the injection of $k_{\langle b\rangle}^{G}$ to $E_{2 p-2}$ with respect to the decomposition above. We set $\delta=\nu \partial: k_{\langle b\rangle}^{G} \longrightarrow K_{2 p-2}$. Then we have the following commutative diagram:

$$
\begin{gathered}
H^{r+2 p-2}\left(G, k_{\langle b\rangle}^{G}\right) \stackrel{\delta_{*}}{\longrightarrow} H^{r+2 p-2}\left(G, K_{2 p-2}\right) \\
\text { Eckmann-Shapiro } \mid \uparrow \operatorname{cor}^{G} \\
H^{r+2 p-2}(\langle b\rangle, k) \stackrel{\psi_{*}}{\longrightarrow} H^{r+2 p-2}\left(\langle b\rangle, K_{2 p-2}\right)
\end{gathered}
$$

where $\psi: k \longrightarrow K_{2 p-2}$ is the $k B$-homomorphism whihc corresponds to the homomorphism $\delta$ under the isomorphism of $\operatorname{Hom}_{k G}\left(k_{\langle b\rangle}^{G}, K_{2 p-2}\right)$ to $\operatorname{Hom}_{k\langle b\rangle}\left(k, K_{2 p-2}\right)$. Since $K_{2 p-2 \mid B}$ is isomorphic with $\Omega^{2(p-1)}\left(k_{B}\right) \oplus$ (projective), we obtain

$$
\begin{array}{cc}
H^{r+2 p-2}\left(B, K_{2 p-2}\right) & \simeq H^{r}(B, k) \\
\operatorname{cor}^{B} \uparrow & \uparrow \operatorname{cor}^{B}=0 \\
H^{r+2 p-2}\left(\langle b\rangle, K_{2 p-2}\right) & \simeq H^{r}(\langle b\rangle, k)
\end{array}
$$

Thus we have

$$
\partial_{*}=0 .
$$

STEP 4. The connecting homomorphism $\omega_{2 p-1}$ is monomorphic and the dimension of its cokernel is 1 .

PROOF. Recall that

$$
E_{2 p-1} \simeq k_{\langle b\rangle}^{G} \text {. }
$$


By our construction one has that the homomorphism $\varkappa: k \longrightarrow E_{2 p-1}$ is not the zero homomorphism. It is enough to show that the induced homomorphism $\partial_{*}$ is the zero homomorphism in the exact cohomology sequence

$$
\begin{aligned}
\cdots \longrightarrow H^{r+2 p-1}(G, k) \stackrel{\mathcal{*} *}{\longrightarrow} H^{r+2 p-1}\left(G, E_{2 p-1}\right) \stackrel{\partial_{*}}{\longrightarrow} H^{r+2 p-1}\left(G, K_{2 p-1}\right) \\
\stackrel{\omega_{2 p-1}}{\longrightarrow} H^{r+2 p}(G, k) \longrightarrow \cdots .
\end{aligned}
$$

Let $\pi: k_{\langle b\rangle}^{G} \longrightarrow k_{\langle b\rangle}^{B}$ and $\theta: k_{\langle b\rangle}^{B} \longrightarrow k_{\langle b\rangle}^{G}$ be the projection and the injection, respectively, with respect to the $k B$-decomposition

$$
k_{\langle b\rangle}^{G}=k_{\langle b\rangle}^{B} \oplus \sum_{1 \neq t \in B \backslash G} k_{\langle b\rangle}^{B} \otimes t .
$$

Then we have

$$
\partial_{*}=\operatorname{res}_{B} \pi_{*} \theta_{*}\left(\partial_{\mid B}\right)_{*} \operatorname{cor}^{G},
$$

as in the proof of Step 2. Hence it is sufficient to verify that

$$
\left(\partial_{B}\right)_{*}=0 .
$$

We have the commutative diagram :

$$
\begin{aligned}
& H^{r+2 p-1}(B, k) \stackrel{\left(\varkappa_{\mid B}\right)_{*}}{\longrightarrow} H^{r+2 p-1}\left(G, k_{\langle b\rangle}^{G}\right) \stackrel{\left(\partial_{B}\right)_{*}}{\longrightarrow} H^{r+2 p-1}\left(B, K_{2 p-1}\right) \\
& \bigoplus_{j=0}^{p-1} \operatorname{res}_{\left\langle b c^{j}\right\rangle} \downarrow \\
& \bigoplus_{j=0}^{p-1} H^{r+2 p-1}\left(\left\langle b c^{j}\right\rangle, k\right) \underset{\nu_{*}}{\stackrel{\sim}{\longrightarrow} \bigoplus_{j=0}^{p-1}} H^{r+2 p-1}\left(\left\langle b c^{j}\right\rangle, k\right)
\end{aligned}
$$

where $\nu$ is the homomorphism which corresponds to the $k B$-homomorphism $\varkappa_{\mid B}: k \longrightarrow \bigoplus_{j=0}^{p-1} k_{\left\langle b c^{j}\right\rangle}^{B}$ under the isomorphism of $\operatorname{Hom}_{k B}\left(k, \bigoplus_{j=0}^{p-1} k_{\left\langle b c^{j}\right\rangle}^{B}\right)$ to $\bigoplus_{j=0}^{p-1} \operatorname{Hom}_{k\left\langle b c^{j}\right\rangle}(k, k)$. By Lemma 3.1 the homomorphism $\bigoplus_{j=0}^{p-1} \operatorname{res}_{\left\langle b c^{j}\right\rangle}$ is epimorphic, and so we have $\left(\partial_{B}\right)_{*}=0$, as desired.

Thus we have established Proposition 3.1.

The following is also needed in Section 4.

LEMMA 3.3. The second cohomology group $H^{2}(M(p), k)$ is four dimensional.

PROOF. This can be verified by the results in the section six in Lewis [15]. But we shall show this lemma by determining a minimal set of generators of the second syzygy $\Omega^{2}(k)$. For $x$ an element in $G$ let $t_{x}$ denote 
the element $(x-1)^{p-1}$ in $k G$. Clearly the first syzygy $\Omega^{1}(k)$ is minimally generated by the elements $a-1$ and $b-1$ in $k G$. Let $\partial: k G \oplus k G \longrightarrow \Omega^{1}(k)$ be the essential epimorphism defined by

$$
(x, y) \longmapsto(a-1) x+(b-1) y \text { for }(x, y) \text { in } k G \oplus k G .
$$

Let us define four elements $w_{1}, w_{2}, w_{3}$, and $w_{4}$ in $k G \oplus k G$ as follows :

$$
\begin{aligned}
& w_{1}=\left(t_{a}, 0\right), w_{2}=\left(0, t_{b}\right) \\
& w_{3}=((a c-1)-b(a-1),(a c-1)(a-1))
\end{aligned}
$$

and

$$
w_{4}=\left(\left(b c^{-1}-1\right)(b-1),\left(b c^{-1}-1\right)-a(b-1)\right) .
$$

It is easily checked that these elements are contained in the kernel $\Omega^{2}(k)$, by using the equality $a b=b a c$. We shall show that the set $\left\{w_{1}, w_{2}, w_{3}, w_{4}\right\}$ is a minimal generating set of $\Omega^{2}(k)$. Since

$$
\begin{aligned}
& w_{1} t_{c}=\left(t_{a} t_{c}, 0\right) \\
& w_{2} t_{c}=\left(0, t_{b} t_{c}\right) \\
& w_{3} t_{c}=\left(-(a-1)(b-1) t_{c},(a-1)^{2} t_{c}\right)
\end{aligned}
$$

and

$$
w_{4} t_{c}=\left((b-1)^{2} t_{c},-(a-1)(b-1) t_{c}\right),
$$

the elements $w_{1}(b-1)^{i}, 0 \leq i \leq p-1, w_{2}(a-1)^{i}, 0 \leq i \leq p-1$, $w_{3}(a-1)^{i}(b-1)^{j}, 0 \leq i \leq p-3,0 \leq j \leq p-2$, and $w_{4}(b-1)^{j}, 0 \leq j \leq p-3$ generate over the group ring $k\langle c\rangle$ a projective $k\langle c\rangle$-module $P$ which is isomorphic with the direct sum of $p^{2}$ copies of $k\langle c\rangle$. And one can verify that

$$
w_{3}(a-1)^{p-2} t_{b}(c-1)^{p-2}=\left(2 a(a-1)^{p-2} t_{b} t_{c}, t_{a} t_{b} t_{c}\right) .
$$

This element is $\langle c\rangle$-invariant and is linearly independent to the $\langle c\rangle$-invariant subspace of the projective $k\langle c\rangle$-module $P$. Thus the $k G$-submodule generated by the elements $w_{1}, w_{2}, w_{3}$, and $w_{4}$ of $k G \oplus k G$ has dimension greater than or equal to $p^{3}+1$. Noting that the dimension of $\Omega^{2}(k)$ is $p^{3}+1$, we see that $\left\{w_{1}, w_{2}, w_{3}, w_{4}\right\}$ is a generating set of $\Omega^{2}(k)$. It is easily seen that no element of $\left\{w_{1}, w_{2}, w_{3}, w_{4}\right\}$ is contained in the submodule generated by the rest of the set. For example if $w_{1}$ is contained in the submodule generated by $w_{2}, w_{3}$, and $w_{4}$, then $w_{1}=w_{2} x+w_{3} y+w_{4} z$ for some $x, y$, and $z$ in $k G$. Applying the element $t_{c}$ to both sides, we have

$$
\begin{aligned}
\left(t_{a} t_{c}, 0\right)= & \left(-(a-1)(b-1) t_{c} y+(b-1)^{2} t_{c} z,\right. \\
& \left.t_{b} t_{c} x+(a-1)^{2} t_{c} y-(a-1)(b-1) t_{c} z\right),
\end{aligned}
$$


a contradiction. The proof is finished.

\section{The period of the periodic module}

The following lemma is our final goal.

LEMMA 4.1. If $n \equiv 1 \bmod p$, then the period of the module $L_{x}$ is $2 p$.

PRoOF. Lemma 2.1 implies that the module $L_{x}$ is periodic. Let $L$ denote the module for short. Restricting the module $L$ to the subgroup $B$, we see that the period of the module $L$ is 2 or $2 p$ by Lemma 4.4 in Benson-Carlson [4]. We must show that the period is not 2. We note that the homomorphism induced by the element $\chi$

$$
x: H^{1}(G, k) \longrightarrow H^{1+2 n}(G, k)
$$

is a monomorphism. In fact for an element $s \lambda+t \mu$ in $H^{1}(G, k)$, where $s$, $t \in k$, we have, letting $A$ denote the maximal subgroup $\langle a, c\rangle$, that

$$
((s \lambda+t \mu) \chi)_{\mid A}=t \mu_{\mid A} f(0) \beta^{n}{ }_{\mid A}
$$

and

$$
((s \lambda+t \mu) \chi)_{\mid B}=s \lambda_{\left|B^{\alpha}\right| B}{ }^{n} .
$$

Hence if $(s \lambda+t \mu) \chi=0$, then both $s$ and $t$ are zero. Therefore, since in the exact cohomology sequence

$$
\begin{aligned}
0 \longrightarrow \operatorname{Hom}_{k G}(k, k) & \longrightarrow \operatorname{Hom}_{k G}\left(\Omega^{2 n}(k), k\right) \longrightarrow \operatorname{Hom}_{k G}(L, k) \\
& \stackrel{\omega}{\longrightarrow} \operatorname{Ext}_{k G}^{1}(k, k) \stackrel{\bar{\chi} *}{\longrightarrow} \operatorname{Ext}_{k G}^{1}\left(\Omega^{2 n}(k), k\right) \longrightarrow \cdots
\end{aligned}
$$

associated with the exact sequence

$$
0 \longrightarrow L \longrightarrow \Omega^{2 n}(k) \stackrel{\hat{\chi}}{\longrightarrow} k \longrightarrow 0
$$

it holds that

$$
\begin{aligned}
\operatorname{Im} \omega & \simeq \operatorname{Ker}(\cdot x) \\
& =0,
\end{aligned}
$$

we obtain the exact sequence

$$
0 \longrightarrow \operatorname{Hom}_{k G}(k, k) \longrightarrow \operatorname{Hom}_{k G}\left(\Omega^{2 n}(k), k\right) \longrightarrow \operatorname{Hom}_{k G}(L, k) \longrightarrow 0 .
$$

This implies that

$$
\operatorname{dim} \operatorname{Hom}_{k G}(L, k)=\operatorname{dim} H^{2 n}(G, k)-1 .
$$

On the other hand since the socle of the module $L$ is isomrphic with 
that of $\Omega^{2 n}(k)$, we see that

$$
\begin{aligned}
\operatorname{dim} \operatorname{Soc}(L) & =\operatorname{dim} \operatorname{Soc}\left(\Omega^{2 n}(k)\right) \\
& =\operatorname{dim} H^{2 n-1}(G, k) .
\end{aligned}
$$

Now suppose that $n \equiv 1 \bmod p$. Then we can put $n=m p+1$. By the dimension formula Proposition 3.1 and Lemma 3.3 we have

$$
\operatorname{dim} H^{2 n}(G, k)=4+2 m(p+1)
$$

and

$$
\operatorname{dim} H^{2 n-1}(G, k)=2+2 m(p+1) .
$$

Thus there exist the following exact sequences:

$$
0 \longrightarrow \Omega(L) \longrightarrow k G^{3+2 m(p+1)} \longrightarrow L \longrightarrow 0
$$

and

$$
0 \longrightarrow L \longrightarrow k G^{2+2 m(p+1)} \longrightarrow \Omega^{-1} \longrightarrow 0 .
$$

Consequently $\Omega(L)$ and $\Omega^{-1}(L)$ are not isomorphic. This proves the lemma.

Acknowledgement. Part of this work was done when the author visited Swiss Federal Institute of Technology Zurich for about a year from March of 1988. The author would like to express his sincere gratitude to Professor U. Stammbach and the staff of the Research Institute of Mathematics in the institute for their kind hospitality. The author also thanks to Professor J. F. Carlson and Professor T. Okuyama for their good suggestions.

\section{References}

[1] J. ALPERIN and L. EVENS, Representations, resolutions and Quillen's dimension theorem, J. Pure Appl. Algebra 22 (1981) pp. 1-9.

[2] J. ALPERIN and L. EVENS, Varieties and elementary abelian groups, J. Pure Appl. Algebra 26 (1982), pp. 221-227.

[ 3 ] D. BENSON, "Modular Representation Theory: New Trends and Methods", Lecture Notes in Mathematics No. 1081, Springer-Verlag, Berlin/Heidelberg/New York/ Tokyo, 1984.

[4] D. BENSON and J. F. CARLSON, Nilpotent elements in the Green ring, J. Algebra 104 (1986), pp. 329-350.

[5] D. BENSON and J. F. CARLSON, Diagramatic methods for modular representations and cohomology, Comm. Algebra 15 (1987) 66. 53-121.

[6] J. F. CARLSON, Periodic modules with large periods, Proc. Amer. Math. Soc. 76 (1979), pp. 209-215.

[7] J. F. CARLSON, The structure of Periodic modules over modular group algebras, J. 
Pure Appl. Algebra 22 (1981), 66. 43-56.

[ 8 ] J. F. CARLSON, Varieties and cohomology ring of a module, J. Algebra 85 (1983), pp. 104-143.

[ 9 ] J. F. CARLSON, The variety of an indecomposable module is connected, Invent. Math. 77 (1984), pp. 291-299.

[10] J. F. CARLSON, “Module Varieties and Cohomology Rings of Finite Groups", Universität Essen, Essen, 1985.

[11] L. Chouinard, Projectivity and relative projectivity over group rings, J. Pure Appl. Algebra 7 (1976), pp. 278-302.

[12] C. CURTIS and I. REINER, “Methods in Representation Theory with Applications to Finite Groups and Orders”, Vol. I, John. Wiler \& Sons, Inc., New York/Toronto, 1981.

[13] L. Evens, A generalization of the transfer map in the cohomology of groups, Trans. Amer. Math. Soc. 108 (1963), pp. 54-65.

[14] R. KNÖRR, Relative projective covers, “Proc. Symp. Modular Representations of Finite Groups”, Aarhus University, Aarhus, 1978, pp. 28-32.

[15] G. LEWIS, The integral cohomology rings of groups of order $p^{3}$, Trans. Amer. Math. Soc. 132 (1968), pp. 501-529.

[16] T. OKUYAma and H. SASAKI, Evens' norm map and Serre's theorem on the cohomology algebra of a p-group, Arch. Mathematik. 54 (1990), pp. 331-339.

[17] T. OKUYAma and H. SASAKI, Periodic modules of large periods for metacyclic p-groups, J. Algebra, to appear.

[18] D. Quillen, A cohomological criterion for p-nilpotence, J. Pure Appl. Algebra 1 (1971), pp. 361-372.

[19] D. QUILLEN and B. VENKOV, Cohomology of finite groups and elementary abelian subgroups, Topology 11 (1972), pp. 317-318.

[20] J. P. SERRE, Sur la dimension cohomologique des groupes profinis, Topology 3 (1965), pp. 413-420.

[21] J. P. SERRE, Une relation dans la cohomologie des p-groupes, C. R. Acad. Sc. Paris, t. 304 , Series I, no. 20 (1987), pp. 587-590.

Faculty of Education Yamaguchi University

Yamaguchi

753 Japan 